\title{
Speeding Up The Wide-pipe: Secure and Fast Hashing
}

\author{
Mridul Nandi ${ }^{\dagger} \quad$ Souradyuti Paul ${ }^{\ddagger}$ \\ ${ }^{\dagger}$ Computer Science Department, C. R. Rao AIMSCS Institute* \\ ${ }^{\ddagger}$ National Institute of Standards and Technology \\ Security Technology Group \\ 100 Bureau Dr., MS 8931 \\ Gaithersburg, MD 20899, United States. \\ ${ }^{\ddagger}$ Katholieke Universiteit Leuven, Dept. ESAT/COSIC, \\ Kasteelpark Arenberg 10, \\ B-3001, Leuven-Heverlee, Belgium. \\ mridul.nandi@gmail.com, souradyuti.paul@nist.gov
}

\begin{abstract}
In this paper we propose a new sequential mode of operation - the Fast wide pipe or FWP for short - to hash messages of arbitrary length. The mode is shown to be (1) preimage-resistance preserving, (2) collision-resistance-preserving and, most importantly, (3) indifferentiable from a random oracle up to $\mathcal{O}\left(2^{n / 2}\right)$ compression function invocations. In addition, our rigorous investigation suggests that any variants of Joux's multi-collision, Kelsey-Schneier 2nd preimage and Herding attack are also ineffective on this mode. This fact leads us to conjecture that the indifferentiability security bound of FWP can be extended beyond the birthday barrier. From the point of view of efficiency, this new mode, for example, is always faster than the Wide-pipe mode when both modes use an identical compression function. In particular, it is nearly twice as fast as the Wide-pipe for a reasonable selection of the input and output size of the compression function. We also compare the FWP with several other modes of operation.
\end{abstract}

\section{Introduction}

A hash function $H:\{0,1\}^{*} \longrightarrow\{0,1\}^{n}$ is a mathematical function which takes as input a binary string of arbitrary length and outputs a binary string of finite length. A secure hash function can be applied in many applications such as data authentication, digital signature, commitment protocols and password protection. A very popular trend of designing a hash function is executing a fixed-input-length (FIL) compression function in a sequential mode as many times as to take the whole message as input. Many practical hash functions, such as MD4 20, MD5 [21, SHA-0 [18], SHA-1 [19] follow the aforementioned design paradigm. These hash functions precisely have two components: (1) a compression function and (2) a mode of operation.

This paper is all about design and analysis of a new hash mode of operation, which is named the Fast wide pipe or FWP for short.

Related work. The classical Merkle-Damgärd mode is the most widely used and most studied hash mode of operation. [17/8. The mode is simple and collision-resistance-preserving ${ }^{1}$ All the practical hash functions mentioned before are based on the Merkle-Damgärd mode. The landscape

* The large part of the work has been done while working in The George Washington University.

${ }^{1}$ In a collision-resistance-preserving hash function collision resistance of a compression function implies collision resistance of the entire hash function. 
Table 1. Comparison among several hash modes of operation with respect to indifferentiability attacks. All numbers are in bits. By Input and Output in the table, we mean bits into and bits out of the compression function.

\begin{tabular}{|c|c|c|c|c|c|c|c|}
\hline Mode & Hash-length & Input & Output & Rate & Lower Bound & Upper Bound & Condition \\
\hline \hline MD[17] & $n$ & $a$ & $b=n$ & $a-b$ & 0 & 0 & $a>b$ \\
\hline MDP[10] & $n$ & $a$ & $b=n$ & $a-b$ & $n / 2$ & $n / 2$ & $a>b$ \\
\hline Wide-pipe[15] & $n$ & $a$ & $b=2 n$ & $a-b$ & $\approx n$ & $\approx n$ & $a>b$ \\
\hline Sponge[2] & $n$ & $a$ & $b=a$ & $a-n$ & $n / 2$ & $n / 2$ & $a>n$ \\
\hline JH[234] & $n$ & $a$ & $b=a$ & $a / 2$ & $n / 3$ & $n$ & $a>2 n$ \\
\hline FWP & $\boldsymbol{n}$ & $\boldsymbol{a}$ & $\boldsymbol{b}=\mathbf{2} \boldsymbol{n}$ & $\boldsymbol{a}-\frac{\boldsymbol{b}}{\mathbf{2}}$ & $\boldsymbol{n} / \mathbf{2}$ & $\boldsymbol{n}$ & $\boldsymbol{a}>\boldsymbol{b} / \mathbf{2}$ \\
\hline
\end{tabular}

is no longer the same. A telltale proof of declining interest of the designers in this mode is that none of the 51 hash functions competing in the ongoing NIST hash function competition uses the classical Merkle-Damgärd mode. The main reasons for discarding this mode by the designers are a few influential attacks: Length extension attack, multi-collision attack [11, Kelsey-Schneier 2nd preimage attack [14] and Herding attack [12. On the positive side, the slow and gradual departure of the classical Merkle-Damgärd hash mode has motivated two new lines of research which go nearly hand in hand: (1) design of new modes of operation and (2) development of new security frameworks to analyze hash functions. The first line of research has indeed resulted in a number of new modes of operation - Wide-pipe [15, HAIFA [5], Sponge [2, EMD 1], JH23] are some of them. One of the major results of the second line of research is the indifferentiability framework developed by Maurer et al. [16. Against this framework, we measure the extent to which a hash function is behaving as a random oracle under a suitable assumption on the underlying compression function. Informally speaking if a hash function is indifferentiable from a random oracle then, for example, it does not come under length extension attack (assuming the underlying compression function is a FIL random oracle). It is, therefore, important that a new mode of operation is both collision-resistance-preserving and indifferentiable from a random oracle. Another crucial issue is to recognize that a hash function indifferentiable from a random oracle does not guarantee that it is collision-resistance-preserving (e.g. modes of operation designed in [7] are not collision-resistancepreserving, although they are indifferentiable from random oracles[1]). These two properties should be analyzed separately [1].

Our contribution. To make a hash function resistant against Joux's multi-collision-type attacks, Lucks has proposed to make the intermediate chaining values of the Merkle-Damgärd mode twice as long as the final hash value; this mode is known as the Wide-pipe mode [15. Suppose the compression function in a Merkle-Damgärd based hash function is defined as $C:\{0,1\}^{m+n} \rightarrow\{0,1\}^{n}$. Lucks has, very rightly, advocated to use a compression function $C:\{0,1\}^{m+2 n} \rightarrow\{0,1\}^{2 n}$ to avoid Joux's multi-collision-type attacks 1113. We call this compression function Lucks' compression function. The message and chaining input to the Lucks' compression function are $m$ and $2 n$ bits. Using any Lucks' compression function $C:\{0,1\}^{m+2 n} \rightarrow\{0,1\}^{2 n}$ we design a hash function FWP, where the message and the chaining input to the compression function are $m+n$ and $n$ bits; we, thus, speed up the hashing operation by allowing $m+n$ bits of message instead of just $m$ bits per compression function invocation. At the same time we prove that the FWP mode is collision- 
resistance preserving and indifferentiable from a random oracle up to $\mathcal{O}\left(2^{n / 2}\right)$ compression function invocations. The fact that the FWP does not come under Joux's multi-collision-type attacks, such as Kelsey-Schneier 2nd preimage attack, leaves open the possibility to extend the indifferentiability bound beyond the birthday barrier.

In Table 1. we compare our results with several other competing hash modes with respect to indifferentiability attacks. Against other attacks such as collision all the modes perform almost identically. It is readily observable that the FWP outperforms all other modes in at least one of the three properties, namely Rate, Lower Bound and Upper Bound in Table 1. The important features of the FWP are pointed out below.

1. FWP performs better than the Wide-pipe with respect to the rate of the hash function. For example, when the input size of the compression function is three times the output size - which is a reasonable choice - FWP is twice faster than the Wide-pipe.

2. Efficiency-wise, FWP has similar performance as Sponge and JH. However, there is a strong evidence that the indifferentiability security bound of FWP can be extended beyond $n / 2$ bits, while there already exists an attack on the Sponge with work factor $n / 2$ bits.

\section{Notation and Convention}

Table 2. Notation

\begin{tabular}{|c|c|}
\hline$\{0,1\} \leq l$ & $\{\varepsilon\} \cup\{0,1\} \cup\{0,1\}^{2} \cup\{0,1\}^{3} \cup \ldots \cup\{0,1\}^{l}$ \\
\hline$[x, y]$ & The set of integers $x, x+1, \ldots, y$ \\
\hline$a|| b$ & concatenation of $a$ and $b$ \\
\hline$|X|$ & Size of set $X ;$ Bit-length if $X$ is a string \\
\hline pad $(M)$ & The sequence of bits after padding $M$ \\
\hline fixed-input-length & Fixed input length \\
\hline variable-input-length & Variable input length \\
\hline FWP & Fast wide-pipe \\
\hline
\end{tabular}

In addition to the above notation, we shall use another set of notation in the context of indifferentiability results of the hash function FWP. They are described in Sect. 5.1.

\section{The New Mode Fast Wide Pipe or FWP}

In this section we define a new sequential mode of operation Fast Wide Pipe (or FWP for short) for hashing messages of length up to $2^{64}$ bits.

Diagrammatic representation of the mode FWP is given in Fig. 1. A four-block example of FWP including a comparison of the new mode with several others is given in Appendix A An algorithmic description is in Algorithm 3.1. The padding $\operatorname{rule} \operatorname{pad}(M)$ is the execution of the following operation: append $t$ zero bits and a 64-bit encoding of $|M|$ to the message $M$. Select the least integer $t \geq 0$ such that $|M|+t+n+64=0 \bmod l$ (see Algorithm 3.1 for the notation). We now make attempts to 


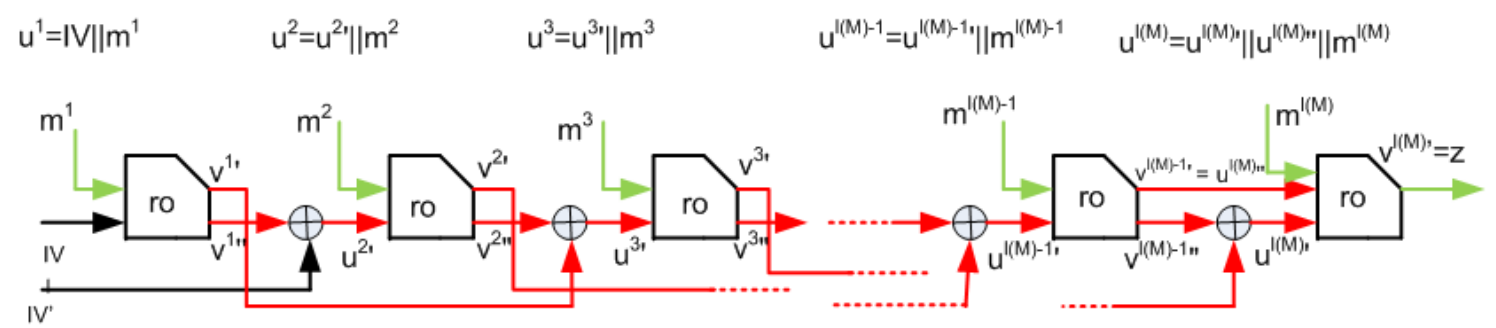

Fig. 1. The new mode FWP. Message $M=m^{1} m^{2} \ldots m^{\ell(M)}$ is hashed by FWPro. The symbols are described in Table 3

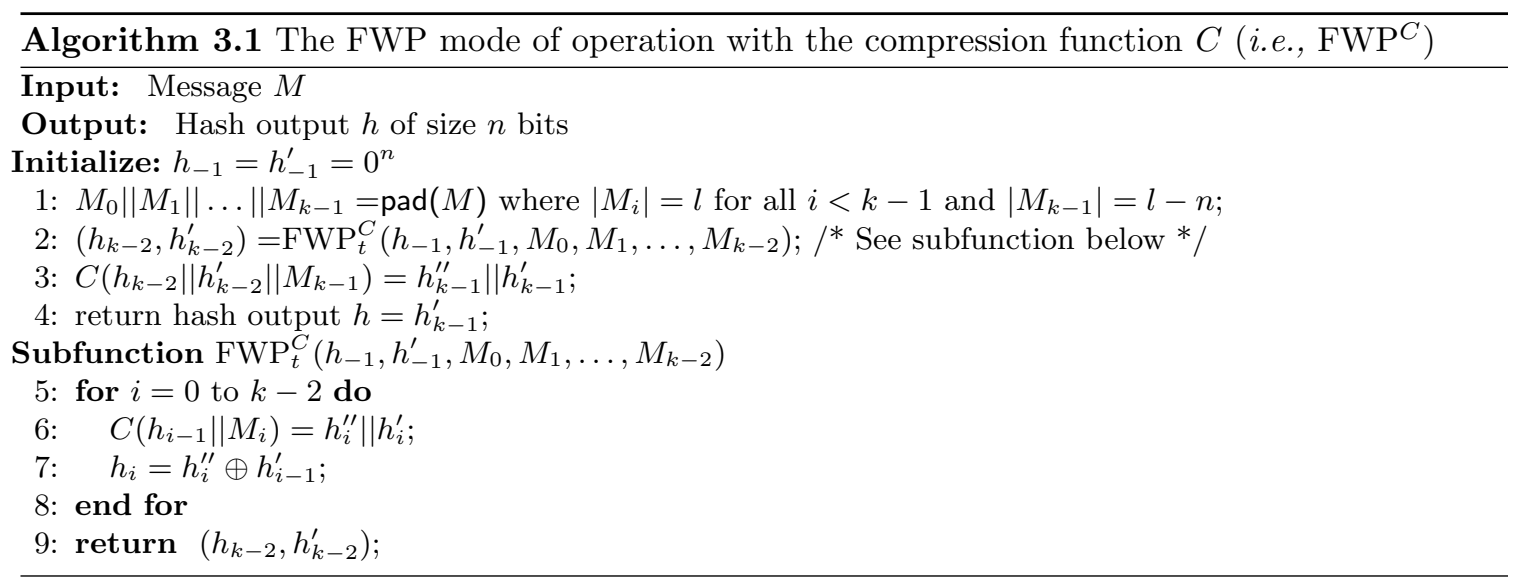

analyze the security of the FWP. For the sake of simplicity, we assume $l-n \geq 64$ which ensures that the length-encoding is completely included in the last block. The entire analysis can be modified easily to include the case $l-n<64$.

\section{Security of the FWP: Resistance Against Collision and Preimage Attacks}

The main results of this section are two theorems which prove that the collision and the preimage attacks on the FWP mode can be reduced to similar attacks on the underlying compression function (see Algorithm 3.1 for the definition of the FWP mode). In other words, the theorems show that finding collision and preimage on the FWP are at least as hard as finding collision and preimage on the compression function.

Before establishing the security results, we first define the following functions. The functions $C_{T}, C_{B}:\{0,1\}^{l+n} \rightarrow\{0,1\}^{n}$ are defined as $C_{T}(x)=h^{\prime}$ and $C_{B}(x)=h^{\prime \prime}$ where $C(x)=h^{\prime \prime} \| h^{\prime}$ (the compression function $C$ of the FWP is defined in Algorithm 3.1).

Theorem 1. If the compression function $C_{T}$ is preimage resistant so is the $F W P^{C}$.

Proof. The theorem can be verified easily by observing the last block of $\mathrm{FWP}^{C}$. 
Glancing at the XOR operations, one may be tempted to conclude that the FWP may be vulnerable against the generalized birthday attack 22. The following theorem drives away such fears.

Theorem 2. If the compression function $C_{T}$ is collision resistant so is the $F W P^{C}$.

Proof. To prove the theorem we need to prove that, if there exists an adversary who finds a pair of messages $\left(M, M^{\prime}\right)$ such that $\mathrm{FWP}^{C}(M)=\mathrm{FWP}^{C}\left(M^{\prime}\right)$ and $M \neq M^{\prime}$ then there exists an adversary who can find $X \neq X^{\prime}$ such that $C_{T}(X)=C_{T}\left(X^{\prime}\right)$.

Suppose an adversary finds a pair $\left(M, M^{\prime}\right)$ such that $\operatorname{FWP}^{C}(M)=\mathrm{FWP}^{C}\left(M^{\prime}\right)$ and $M \neq M^{\prime}$. Now there are two possible cases.

CASE 1: $|M| \neq\left|M^{\prime}\right|$. Suppose that the number of message-blocks in $\operatorname{pad}(M)$ and $\operatorname{pad}\left(M^{\prime}\right)$ are $a$ and $b$ where $a \neq b$. Note, as per our definition of $C$ and $\mathrm{FWP}^{C}, M_{a-1} \neq M_{b-1}^{\prime}$ due to the length padding. Now, $\operatorname{FWP}^{C}(M)=\mathrm{FWP}^{C}\left(M^{\prime}\right)$ implies $C_{T}\left(h_{a-2}\left\|h_{a-2}^{\prime}\right\| M_{a-1}\right)=C_{T}\left(g_{b-2}\left\|g_{b-2}^{\prime}\right\| M_{b-1}^{\prime}\right)$. Therefore, we get a collision on $C_{T}$.

CASE 2: $|M|=\left|M^{\prime}\right|$. Suppose that the number of message-blocks in $\operatorname{pad}(M)$ is $a$. Now there are two cases.

CASE 2(A): $C_{T}\left(h_{a-2}\left\|h_{a-2}^{\prime}\right\| M_{a-1}\right)=C_{T}\left(g_{a-2}\left\|g_{a-2}^{\prime}\right\| M_{a-1}^{\prime}\right)$

where $h_{a-2}\left\|h_{a-2}^{\prime}\right\| M_{a-1} \neq g_{a-2}\left\|g_{a-2}^{\prime}\right\| M_{a-1}^{\prime}$. Therefore, we obtain a collision on $C_{T}$.

CASE 2(в): $C_{T}\left(h_{a-2}\left\|h_{a-2}^{\prime}\right\| M_{a-1}\right)=C_{T}\left(g_{a-2}\left\|g_{a-2}^{\prime}\right\| M_{a-1}^{\prime}\right)$

where $h_{a-2}\left\|h_{a-2}^{\prime}\right\| M_{a-1}=g_{a-2}\left\|g_{a-2}^{\prime}\right\| M_{a-1}^{\prime}$.

The above equation implies that $\operatorname{FWP}_{t}^{C}\left(0^{n}\left\|0^{n}\right\| M_{0}\|\ldots\| M_{a-2}\right)=\mathrm{FWP}_{t}^{C}\left(0^{n}\left\|0^{n}\right\| M_{0}^{\prime}\|\ldots\| M_{a-2}^{\prime}\right)$ which in turn implies collision on $C_{T}$ by Lemma 1 (the definition of $\mathrm{FWP}_{t}^{C}$ is provided in Algorithm 3.1). Now the only remaining part needed to complete the proof is the proof of Lemma 1 which is provided below.

The following lemma has been used in Theorem 2 It will be further used to obtain some indifferentiability results of the $\mathrm{FWP}^{C}$ in Sect. 5

Lemma 1. If the compression function $C_{T}$ is collision resistant then the $F W P_{t}^{C}$ is free-start collision resistant for fixed length messages. In other words, if there exists an adversary who finds two triples $\left(h_{-1}, h_{-1}^{\prime}, M\right) \neq\left(g_{-1}, g_{-1}^{\prime}, M^{\prime}\right)$ such that $|M|=\left|M^{\prime}\right|(|M|$ is a multiple of $l)$ and $F W P_{t}^{C}\left(h_{-1}, h_{-1}^{\prime}, M\right)=F W P_{t}^{C}\left(g_{-1}, g_{-1}^{\prime}, M^{\prime}\right)$, then there exists an adversary who finds $X \neq X^{\prime}$ such that $C_{T}(X)=C_{T}\left(X^{\prime}\right)$.

Proof. Suppose there exists an adversary who finds two triples $\left(h_{1}, h_{1}^{\prime}, M\right) \neq\left(g_{1}, g_{1}^{\prime}, M^{\prime}\right)$ such that $|M|=\left|M^{\prime}\right|$ (the number of message-blocks in $M$ is $a$ ) and $\mathrm{FWP}_{t}^{C}\left(h_{-1}, h_{-1}^{\prime}, M\right)=\mathrm{FWP}_{t}^{C}\left(g_{-1}, g_{-1}^{\prime}, M^{\prime}\right)$. In order to obtain a pair $X \neq X^{\prime}$ such that $C_{T}(X)=C_{T}\left(X^{\prime}\right)$ we need to check at most $a$ equations whether they are satisfied:

$$
C\left(h_{i-1}, M_{i}\right) \stackrel{?}{=} C\left(g_{i-1}, M_{i}^{\prime}\right) \text { where } i=a-1, \ldots, 0 .
$$

We claim that the above verification will produce some $m$ with $0 \leq m \leq a-1$ such that $C_{T}\left(h_{m-1}, M_{m}\right)=C_{T}\left(g_{m-1}, M_{m}^{\prime}\right)$ and $\left(h_{m-1}, M_{m}\right) \neq\left(g_{m-1}, M_{m}^{\prime}\right)$ and thus, the lemma is proved. This claim can be proved by the following crucial observation on $\mathrm{FWP}_{t}^{C}$.

Observation: For all $i \in[0, a-1],\left(h_{i}, h_{i}^{\prime}\right)=\left(g_{i}, g_{i}^{\prime}\right)$ implies one of the following two statements:

(1) $\left(h_{i-1}, M_{i-1}\right) \neq\left(g_{i-1}, M_{i-1}^{\prime}\right)$ which implies collision on $C_{T}$,

(2) $\left(h_{i-1}, M_{i-1}\right)=\left(g_{i-1}, M_{i-1}^{\prime}\right)$ which implies $\left(h_{i-1}, h_{i-1}^{\prime}\right)=\left(g_{i-1}, g_{i-1}^{\prime}\right)$.

Next, we move on to analyze the FWP in a different security framework known as the indifferentiability framework. 


\section{Security of the FWP Mode: Indifferentiable From a Random Oracle}

In this section we discuss the indifferentiability property of the FWP mode. In the context of hash function, an important use of the indifferentiability framework developed by Maurer et al. [16] is the determination of whether a variable-input-length hash function behaves reasonably randomly when the underlying compression function is a fixed-input-length random oracle. There is a considerable chance for the reader to be lured into believing that the collision resistance preservation (described in Sect. 4) and the indifferentiability property of a hash function may be related. In particular, one may be inclined to intuiting that one property implies the other. Such intuition is not true [1]. These two properties are orthogonal and need to be analyzed separately.

\subsection{Preliminaries: Introduction to Indifferentiability Framework}

We begin with the definition of a random oracle; this useful object will be used frequently in the subsequent discussion.

Definition 1 (Random oracle). A random oracle is a function $R O: X \rightarrow Y$ chosen uniformly at random from the set of all $|Y|^{|X|}$ functions that map $X \rightarrow Y$. In other words, a function $R O: X \rightarrow Y$ is a random oracle if and only if, for each $x \in X$, the $R O(x)$ is chosen uniformly at random from $Y$.

Corollary 1. If a function $R O: X \rightarrow Y$ is a random oracle then

$$
\operatorname{Pr}\left[R O(x)=y \mid R O\left(x_{1}\right)=y_{1}, R O\left(x_{2}\right)=y_{2}, \ldots, R O\left(x_{q}\right)=y_{q}\right]=\frac{1}{|Y|}
$$

where $x \notin\left\{x_{1}, x_{2}, \ldots, x_{q}\right\}, y \in Y$ and $q \in \mathbb{Z}$.

Now we introduce the indifferentiability framework and briefly discuss its significance. The following definition is a slightly modified version of the original definition provided in [16].

Definition 2 (Indifferentiability framework). [16] A Turing machine $T$ with oracle access to an ideal primitive $\mathcal{F}$ is said to be $\left(t_{\mathcal{A}}, t_{S}, q, \sigma, \varepsilon\right)$-indifferentiable from an ideal primitive $\mathcal{G}$ if there exists a simulator $S$ such that for any distinguisher $\mathcal{A}$ the following equation is satisfied:

$$
\operatorname{Adv}_{\mathcal{A}}((T, \mathcal{F}),(\mathcal{G}, S))=\left|\operatorname{Pr}\left[\mathcal{A}^{T, \mathcal{F}}=1\right]-\operatorname{Pr}\left[\mathcal{A}^{\mathcal{G}, S}=1\right]\right|<\varepsilon
$$

The simulator $S$ is an interactive algorithm which has oracle access to $\mathcal{G}$ and runs in time at most $t_{S}$. The distinguisher $\mathcal{A}$ runs in time at most $t_{\mathcal{A}}$ and makes at most q queries. The total message blocks queried by $\mathcal{A}$ is at most $\sigma$.

Briefly, the significance of indifferentiability property is described as follows: Suppose, an ideal primitive $\mathcal{G}$ (e.g. a variable-input-length random oracle) is indifferentiable from an algorithm $T$ based on another ideal primitive $\mathcal{F}$ (e.g. a fixed-input-length random oracle). In such case, any cryptographic system $\mathcal{P}$ based on $\mathcal{G}$ is as secure as the $\mathcal{P}$ based on $T^{\mathcal{F}}$ (i.e., $T^{\mathcal{F}}$ replaces $\mathcal{G}$ in $\mathcal{P}$ ). See [16] for more on that.

Pictorial Description of Def. 2(Fig. 2). In the figure, five entities involved in Def. 2 are shown with an example. Suppose, the oracle Turing machine $T$, the ideal primitives $\mathcal{F}, \mathcal{G}$ are, respectively, a hash function $H$, random oracles ro and RO. The exchange of queries and responses is also shown in the figure. Note that it is forbidden to issue queries in the opposite directions. For example, the hash function $H$ can send a query to ro and receive response, but never the other way round. In this setting, Def. 2 addresses the degree to which any computationally bounded adversary is unable to distinguish between Option 1 and Option 2. 


\subsection{Indifferentiability Framework for FWP: Designing a Simulator $S$}

In this section we describe the entities of Fig. 2 with respect to the hash function FWP: $\{0,1\} \leq 2^{64} \rightarrow$ $\{0,1\}^{n}$. The mode FWP is defined in Fig. 11. In the rest of the paper the $H$ is understood to be the FWP hash function. The fixed-input-length random oracle ro : $\{0,1\}^{r+n} \rightarrow\{0,1\}^{2 n}$ is the compression function invoked by the FWP mode. The variable-input-length random oracle RO is defined as RO : $\{0,1\} \leq 2^{64} \rightarrow\{0,1\}^{n}$. The only remaining part to complete the indifferentiability framework is designing a simulator $S$. This section is devoted to that. The fifth entity of Fig. 2 , which is an arbitrary distinguisher $\mathcal{A}$, is discussed in Sect. 5.3. We kick off with the notation.

Notation. Table 3 provides the notation useful to follow our indifferentiability results on the new hash function FWP. Note that the notation can be very easily adapted to any hash function based on a sequential mode of operation.

Now we define a few terms - in relation to Fig. 1 and 2 - which will be used to arrive at our main indifferentiability results of Sect. 5.3 .

Queries and lists. We now define various types of queries and lists (or arrays) that can potentially be used by a distinguisher to separate a hash function from a random oracle. The first assumption is that a distinguisher does not resubmit to an oracle a query whose response is already known. This is a valid assumption because, in our case, an identical oracle - any of FWP hash function, ro, RO and $S$ of Fig. 2 - gives identical response to an identical query (it would be further clear when we shall concretely define the simulator $S$ ). Our next assumption is that, unless otherwise specified, a query is known to be submitted by the distinguisher. In the present case, we are not interested in queries submitted by the simulator $S$ or by the hash function FWP. Now we define two special types of queries.

Definition 3 (Short and long query). A query submitted to $S$ or ro is defined as a short query. Similarly, a query submitted to FWP or RO is defined as the long query (see Fig. 2).

At this time it is important to discuss a subclass of short and long queries known as trivial queries. For easy understanding, we try to introduce the notion without the rigors of mathematical notation as much as possible; however, our treatment is logically sound and foolproof. The motivation behind the determination of trivial queries is that their outputs are implied by the previous queries and their responses, no matter whether the distinguisher is interacting with Option 1 or Option 2 of

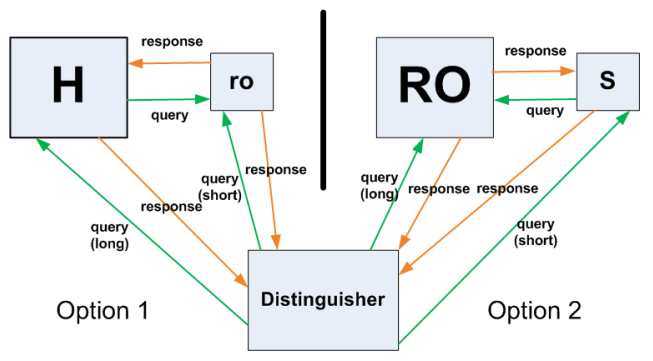

Fig. 2. The entities and their behavior involved in the indifferentiability framework of Def. 2. $T \equiv H$, $\mathcal{F} \equiv$ ro, $\mathcal{G} \equiv \mathrm{RO}, S \equiv$ simulator (see description above). In Sect. 5.2, $H$ is the FWP hash function.

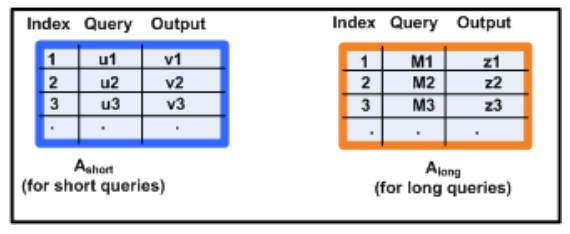

Fig. 3. Several databases maintained by the distinguisher 
Table 3. The notation used in the indifferentiability framework for FWP (see Fig. 1)

\begin{tabular}{|c|c|c|}
\hline Symbol & bit-length & Description \\
\hline$A_{\text {short }}, A_{\text {long }}$ & - & Current query-response arrays \\
\hline$A_{\text {inter }}$ & - & Array for intermediate query-responses \\
\hline$A[i, i-1, \ldots j]$ & - & Array (or bit-string) $A$ truncated between indices $i$ and $j$ \\
\hline $\mathcal{A}$ & - & A distinguisher \\
\hline $\mathcal{A}^{\prime}$ & - & Modification of the distinguisher $\mathcal{A}$ \\
\hline$\ell(M)$ & - & Number of compression function calls to hash $M$ \\
\hline$\lambda$ & 0 & Empty String \\
\hline$M$ & $\leq 2^{64}$ & Message $M=m^{1} m^{2} \ldots m^{\ell(M)}$ \\
\hline$m^{k}, m^{\ell(M)}$ & $r, r-n$ & Messages of $k$ th and $\ell(M)$ th compression functions $\left(k<m^{\ell(M)}\right)$ \\
\hline MesgVer & - & Message verification algorithm \\
\hline MesgRecon & - & Message reconstruction algorithm \\
\hline$q, \sigma$ & - & Maximum number of queries and blocks used by distinguisher \\
\hline ro, $\mathrm{RO}$ & - & Random oracles \\
\hline $\mathcal{S}$ & - & Set of reconstructed messages given a short query \\
\hline$S$ & - & The simulator \\
\hline$t_{\mathcal{A}}, t_{S}$ & - & Time of $\mathcal{A}$ and $S$ \\
\hline$u^{k \prime}$ & $n$ & Chaining input to $k$ th compression function $\left(k<m^{\ell(M)}\right)$ \\
\hline$u^{\ell(M) \prime \prime} \| u^{\ell(M) \prime}$ & $2 n$ & Chaining input to $\ell(M)$ th compression function \\
\hline$u^{k}, u^{\ell(M)}$ & $r+n, r+n$ & Total input to $k$ th and $\ell(M)$ th compression functions \\
\hline$v^{k \prime}, v^{k \prime \prime}$ & $n, n$ & Two halves of output from $k$ th compression function \\
\hline$v^{k}$ & $2 n$ & Total output from $k$ th compression function \\
\hline$z$ & $n$ & Final hash value \\
\hline
\end{tabular}


Fig. 2. Therefore, trivial queries cannot be used to distinguish between two systems, even if they satisfy specific 'bad' conditions. Before we formally define trivial queries, some discussion on the databases maintained by the distinguisher and two special functions MesgVer and MesgRecon are necessary. We first discuss them briefly.

Databases of the distinguisher. Let us assume that a distinguisher uses two arrays: (1) $A_{\text {short }}$ for storing short queries and the responses, and (2) $A_{\text {long }}$ for long queries and the responses (see Fig. 31). Queries and their responses are indexed by the time they are submitted. Note that the simulator $S$ can access $\mathrm{A}_{\text {short }}$ but not $\mathrm{A}_{\text {long. }}$.

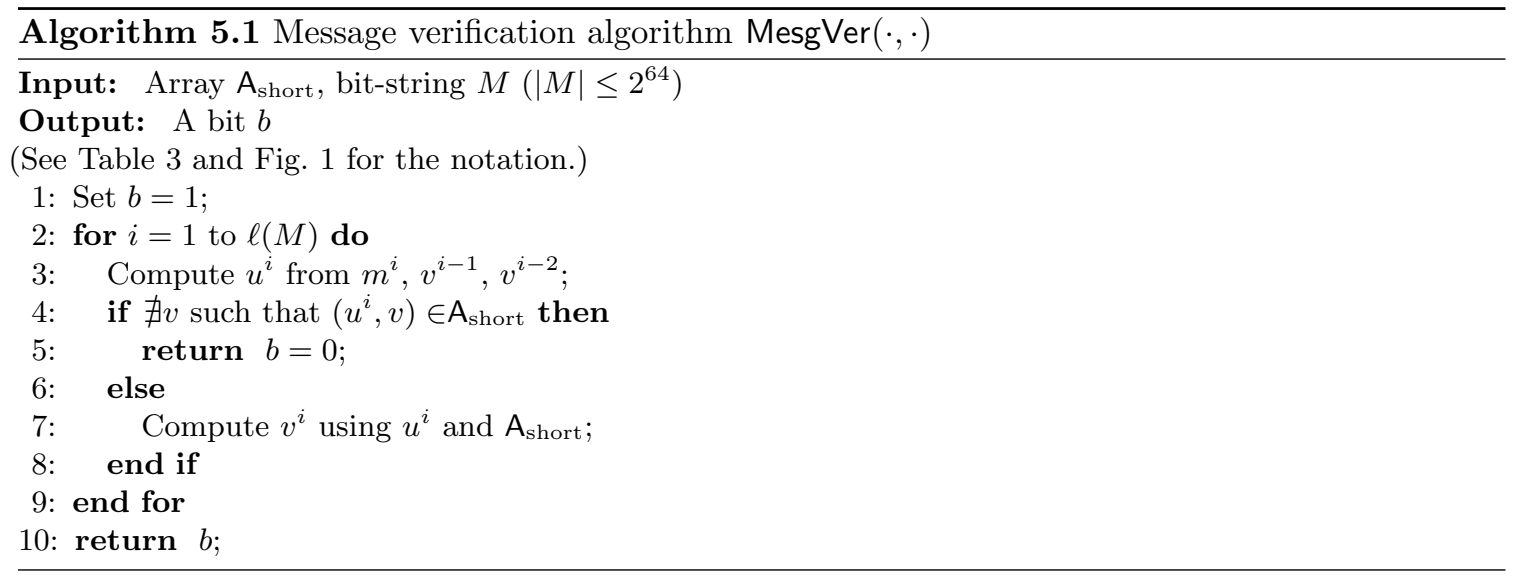

Discussion on algorithms MesgVer and MesgRecon. Informally speaking, MesgVer is a function which takes two inputs - the current list $\mathrm{A}_{\text {short }}$, a long query $M$ - to verify whether the long query $M$ is a valid message for the hash mode FWP. What it essentially does is compute all compression function inputs $-u^{1}, u^{2}, \ldots, u^{\ell(M)}$ - sequentially and checks whether they exist in $\mathrm{A}_{\text {short }}$. The MesgVer algorithm has been described in Algorithm 5.1

The MesgRecon algorithm, in some sense, works in the opposite direction. It takes the current list $\mathrm{A}_{\text {short }}$ and a short query $x$ as inputs and reconstructs a set of messages $\mathcal{S}$ such that each message $M \in \mathcal{S}$ is a valid message for FWP mode and, moreover, the input to the last compression function is $x$. The algorithm is described in Algorithm 5.2.

Now we are ready to define the trivial queries.

Definition 4 (Trivial short query). A short query $x$ is a trivial short query if the following conditions hold:

- MesgRecon $\left(A_{\text {short }}, x\right)=\{M\}$.

- The $M$ has been queried previously as a long query (i.e. $\exists v$ such that $\left.(M, v) \in A_{\text {long }}\right)$.

Definition 5 (Trivial long query). A long query $M$ is a trivial long query if the following conditions hold:

- MesgVer $\left(A_{\text {short }}, M\right)=1$. Suppose the final input $u^{\ell(M)}$ computed in $\operatorname{Mesg} \operatorname{Ver}\left(A_{\text {short }}, M\right)$ is the ith query in $A_{\text {short }}$.

- MesgRecon $\left(A_{\text {short }}[i-1, \ldots, 2,1], u^{\ell(M)}\right)=\{M\}$. 


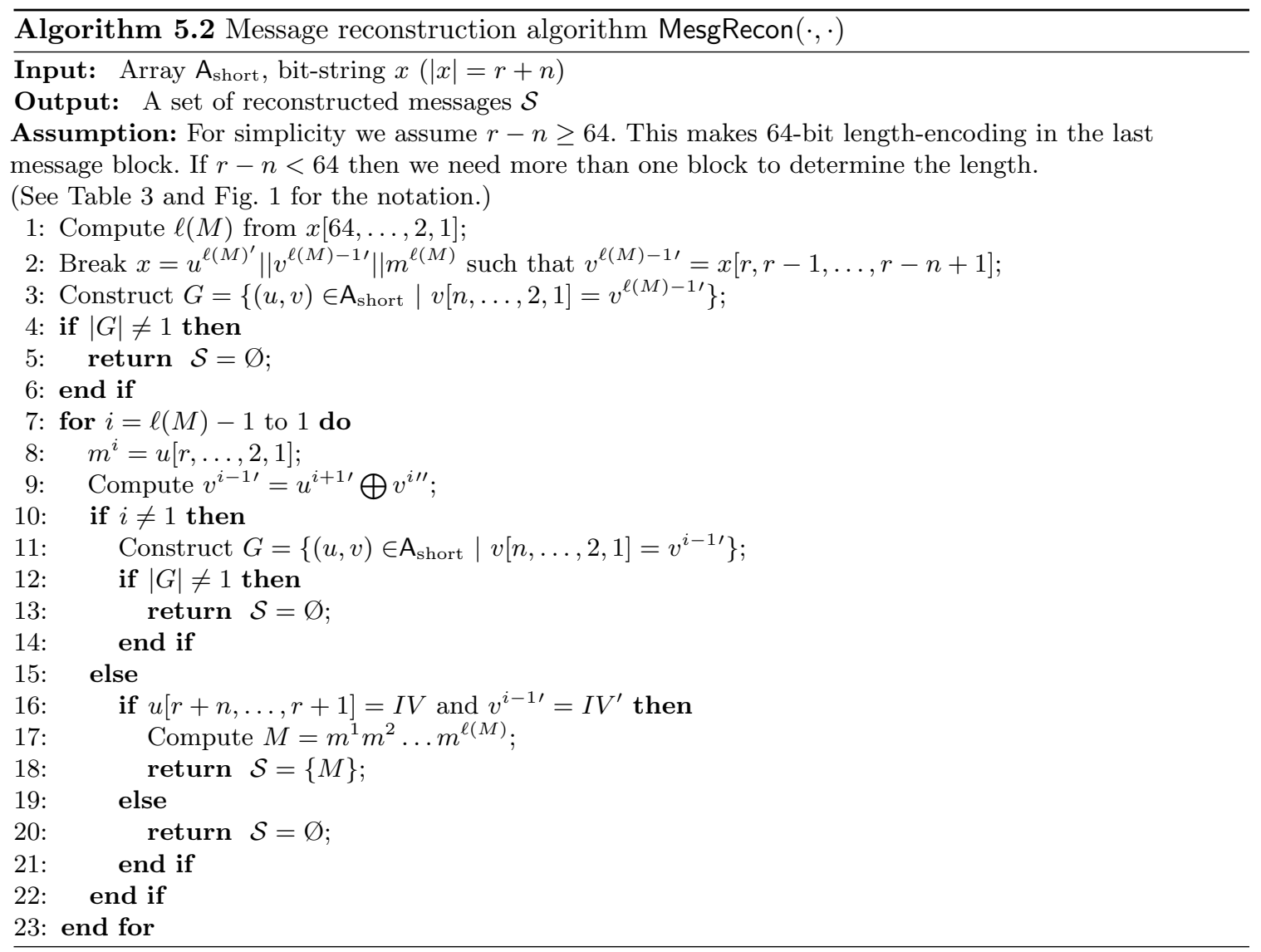


The nontrivial short and long queries are obvious from the above definitions.

Definition 6 (Nontrivial queries). A short query $x$ is a nontrivial short query if it is not a trivial short query. Similarly, a long query $M$ is a nontrivial long query if it is not a trivial long query.

At this point it is useful to, once more, remember the motivation behind separating the trivial queries from all queries. The distinguisher may communicate with (FWP, ro) or (RO, $S$ ). Irrespective of whether it is communicating with (FWP, ro) or $(\mathrm{RO}, S)$, the responses of the trivial queries should be implied by the previous query-responses. Therefore, the trivial queries do not help a distinguisher to differentiate between (FWP, ro) and $(\mathrm{RO}, S)$ (see Fig. 2). We have just concretely defined the trivial queries in Def. 4and 5 . However, we still cannot say whether the trivial queries indeed fulfil the motivation until we prove the existence of a compatible simulator. Such a simulator $S$ is described below.

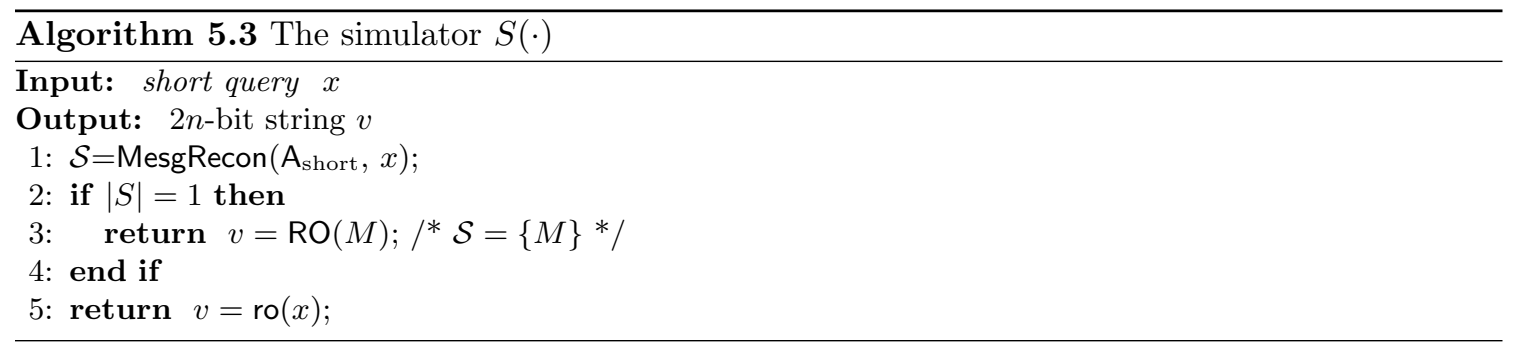

Our design of indifferentiability framework is now complete, except establishing a property that shows, under trivial queries, both (FWP, ro) and (RO, $S$ ) behave identically, if they are supplied with identical $A_{\text {short }}$ and $A_{\text {long. }}$. We capture this property in the following lemma.

Lemma 2. Suppose, for a distinguisher $\mathcal{A}$, the lists $A_{\text {short }}$ and $A_{\text {long }}$ are identical for both ( $F W P$, ro) and $(R O, S)$ after the ith query. Then the following statements are true.

1. If $M$ is the $(i+1)$ th trivial long query then the probability distributions of $F W P^{r o}(M)$ and $R O(M)$ are identical.

2. If $x$ is the $(i+1)$ th trivial short query then the probability distributions of $S(x)$ and ro $(x)$ are identical.

Proof. The proof is immediate from the construction of the simulator $S$ which is described in Algorithm 5.3 .

\subsection{Bounding the Advantage of an Arbitrary Distinguisher}

After designing the simulator $S$ in the previous section, now we are left with the most important part of the paper: to compute an $\varepsilon$ as a function of $\left(t_{\mathcal{A}}, t_{S}, q, \sigma\right)$ (see Def. 2). To that end, we first design an arbitrary oracle algorithm $\mathcal{A}$ (see Algorithm B.1 in Appendix B that separates (FWP, ro) from $(\mathrm{RO}, S)$.

Algorithm B.1 is characterized by two functions: (1) the $f_{\text {query }}(\cdot, \cdot)$ which computes the next query, and $(2)$ the $f_{\text {cond }}(\cdot, \cdot)$ which decides whether the system is (FWP, ro) or (RO, $S$ ). Both the 
functions take the arrays $\mathrm{A}_{\text {short }}, \mathrm{A}_{\text {long }}$ as inputs. To bound the advantage of $\mathcal{A}$, we slightly modify $\mathcal{A}$ to design $\mathcal{A}^{\prime}$ which is described in Algorithm B.2 of Appendix B. We now discuss the algorithms briefly.

Discussion on Algorithm B.1 and B.2, Both $\mathcal{A}$ and $\mathcal{A}^{\prime}$ have identical query function $f_{\text {query }}$. We only modify $f_{\text {cond }}$ of $\mathcal{A}$ to design $f_{\text {cond }}^{\prime}$ of $\mathcal{A}^{\prime}$. The additional parts of $\mathcal{A}^{\prime}$ are placed within boxes in Algorithm B.2. The algorithm $\mathcal{A}^{\prime}$, in addition to $\mathrm{A}_{\text {short }}$ and $\mathrm{A}_{\text {long }}$, uses an extra array $\mathrm{A}_{\text {inter }}$ which, using a function MesgDecom $\left(M_{i}\right)$, stores all intermediate inputs and outputs for any long query $M_{i}$ applied to FWP. Our main task is to define a suitable $f_{\text {cond }}^{\prime}$ such that the following inequality holds:

$$
\max _{\mathcal{A}}|\operatorname{Pr}[\mathcal{A}(\mathrm{FWP}, \mathrm{ro})=1]-\operatorname{Pr}[\mathcal{A}(\mathrm{RO}, S)=1]| \leq \max _{\mathcal{A}^{\prime}} \operatorname{Pr}\left[\mathcal{A}^{\prime}(\mathrm{FWP}, \mathrm{ro})=1\right]
$$

where the maximum values of the right hand side and the left hand side are based on the suitable choices of (1) $f_{\text {query }}$ and $f_{\text {cond }}$, and (2) $f_{\text {cond }}^{\prime}$ respectively. It is easy to show that he above inequality implies $\operatorname{Adv}_{\mathcal{A}}((\mathrm{FWP}, \mathrm{ro}),(\mathrm{RO}, S)) \leq \max _{\mathcal{A}} \operatorname{Pr}\left[\mathcal{A}^{\prime}(\mathrm{FWP}, \mathrm{ro})=1\right]$. We now define a suitable $f_{\text {cond }}^{\prime}$ recursively.

Definition 7 ( $f_{\text {cond }}^{\prime}$ of Algorithm B.2). The definition is divided into two complementary parts. (1) Let the ith query computed by $f_{\text {query }}$ of $\mathcal{A}^{\prime}$ be a nontrivial long query denoted by $M_{i}$. Then $f_{\text {cond }}^{\prime}=1$ if one or more following conditions are satisfied.

- Collision between the final input for the current long query $M_{i}$ and the final input for some previous long query $M_{j}$. That is, $u_{i}^{\ell\left(M_{i}\right)}=u_{j}^{\ell\left(M_{j}\right)}$ for some $j<i$.

- Collision between the final input for the current long query $M_{i}$ and some intermediate input for some previous long query $M_{j}$. That is, $u_{i}^{\ell\left(M_{i}\right)}=u_{j}^{k}$ for some $j \leq i$ and $k<\ell\left(M_{j}\right)$.

- Collision between some intermediate input for the current long query $M_{i}$ and the final input for some previous long query $M_{j}$. That is, $u_{i}^{k}=u_{j}^{\ell\left(M_{j}\right)}$ for some $j<i$ and $k<\ell\left(M_{i}\right)$.

- Collision between the final input for the current long query $M_{i}$ and some previous short query $x_{j}$. That $i s, u_{i}^{\ell\left(M_{i}\right)}=x_{j}$ for some $j<i$.

Otherwise $f_{\text {cond }}^{\prime}=0$.

(2) Let the ith query computed by $f_{\text {query }}$ of $\mathcal{A}^{\prime}$ be a nontrivial short query denoted by $x_{i}$. Then $f_{\text {cond }}^{\prime}=1$ if the following condition is satisfied.

- Collision between the current short query $x_{i}$ and the final input for some previous long query $M_{j}$. That is, $x_{i}=u_{j}^{\ell\left(M_{j}\right)}$ for some $j<i$.

Otherwise $f_{\text {cond }}^{\prime}=0$.

Now we state the following theorem.

Theorem 3. Under Def. 团 of $f_{\text {cond }}^{\prime}$ the following inequality holds.

$$
\operatorname{Adv}_{\mathcal{A}}((F W P, r o),(R O, S)) \leq \max _{\mathcal{A}^{\prime}} \operatorname{Pr}\left[\mathcal{A}^{\prime}(F W P, r o)=1\right]
$$

Proof. The theorem has been proved for a general domain extension in [3. Note that, in the present case, the event $\mathcal{A}^{\prime}(\mathrm{FWP}$, ro $)=1$ is also an event invoked by $\mathcal{A}(\mathrm{FWP}$, ro $)$ according to Def. 7 - exactly this event has been termed a Bad event for a GDE in [3]. So by using Theorem 1 of [3] we have our result. 
In the remainder of the section we strive to obtain an upper bound $\varepsilon$ on $\max _{\mathcal{A}^{\prime}} \operatorname{Pr}\left[\mathcal{A}^{\prime}(\mathrm{FWP}, \mathrm{ro})=1\right]$. According to Theorem 3, $\varepsilon$ is an upper bound on $\mathbf{A d v}_{\mathcal{A}}((\mathrm{FWP}, \mathrm{ro}),(\mathrm{RO}, S))$ too.

We have two databases $A_{\text {short }}$ and $A_{\text {inter }}$ which essentially store all invocations to ro. Each element of $A_{\text {short }}$ and $A_{\text {inter }}$ is of the form $(u, v)$ where $u \in\{0,1\}^{r+n}$ and $v \in\{0,1\}^{2 n}$. We denote the $i$ th pair by $\mathrm{A}_{\text {short }}(i)=\left(\mathrm{A}_{\text {short }}(i, 1), \mathrm{A}_{\text {short }}(i, 2)\right)$ and $\mathrm{A}_{\text {inter }}(i)=\left(\mathrm{A}_{\text {inter }}(i, 1), \mathrm{A}_{\text {inter }}(i, 2)\right)$.

Whenever we add a pair $(u, v)$ to $\mathrm{A}_{\text {inter }}$ it corresponds to a pair $(M, i)$ such that when we compute $F W P^{\mathrm{ro}}(M)$, the $i$ th intermediate input, output are $u$ and $v$ respectively. Note, when $i=\ell(M) F W P^{\text {ro }}(M)=v[2 n, 2 n-1, \ldots n+1]$.

We define the following bad events. It mainly considers one of the following cases: (1) the unexpected collisions in the first or last half of the outputs of ro which are stored in one of the two databases $\mathrm{A}_{\text {short }}$ and $\mathrm{A}_{\text {inter }}$ during query-responses of $\mathcal{A}^{\prime}$ and (2) collision on the least significant $n$ bits of inputs of ro stored in $\mathrm{A}_{\text {inter }}$ with least significant $n$ bits of inputs of ro stored in one of the two lists.

1. Type-1 bad. $\mathrm{A}_{\text {short }}$ vs. $\mathrm{A}_{\text {short }}$ for output collision: If $\mathrm{A}_{\text {short }}(i, 2)[n, n-1, \ldots 1]=\mathrm{A}_{\text {short }}\left(i^{\prime}, 2\right)[n, n-$ $1, \ldots 1]$ or $\mathrm{A}_{\text {short }}(i, 2)[2 n, 2 n-1, \ldots n+1]=\mathrm{A}_{\text {short }}\left(i^{\prime}, 2\right)[2 n, 2 n-1, \ldots n+1]$ for some $i \neq i^{\prime}$.

2. Type-2 bad. $\mathrm{A}_{\text {short }}$ vs. $\mathrm{A}_{\text {inter }}$ for output collision: If $\mathrm{A}_{\text {short }}(i, 2)[n, n-1, \ldots 1]=\mathrm{A}_{\text {inter }}\left(i^{\prime}, 2\right)[n, n-$ $1, \ldots 1]$ or $\mathrm{A}_{\text {short }}(i, 2)[2 n, 2 n-1, \ldots n+1]=\mathrm{A}_{\text {inter }}\left(i^{\prime}, 2\right)[2 n, 2 n-1, \ldots n+1]$ for some $i, i^{\prime}$ such that the following is not true:

$\mathrm{A}_{\text {inter }}\left(i^{\prime}, 2\right)$ corresponds to the pair $(M, j)$ and the computation of $F W P^{\text {ro }}(M)$ up to $j-1$ intermediate input is already in the list $\left\{\mathrm{A}_{\text {short }}(r): r \leq j-1\right\}$ and the $j^{\text {th }}$ intermediate input is $\mathrm{A}_{\text {inter }}\left(i^{\prime}, 2\right)$.

3. Type-3 bad. $\mathrm{A}_{\text {inter }}$ vs. $\mathrm{A}_{\text {inter }}$ for output collision: If $\mathrm{A}_{\text {short }}(i, 2)[n, n-1, \ldots 1]=\mathrm{A}_{\text {inter }}\left(i^{\prime}, 2\right)[n, n-$ $1, \ldots 1]$ or $\mathrm{A}_{\text {short }}(i, 2)[2 n, 2 n-1, \ldots n+1]=\mathrm{A}_{\text {inter }}\left(i^{\prime}, 2\right)[2 n, 2 n-1, \ldots n+1]$ for some $i, i^{\prime}$ such that the pairs corresponding to $\mathrm{A}_{\text {short }}(i, 2)$ and $\mathrm{A}_{\text {inter }}\left(i^{\prime}, 2\right)$ are not identical.

4. Type-4 bad. $\mathrm{A}_{\text {inter }}$ vs. both list for input collision: $\mathrm{A}_{\text {inter }}(i, 1)[n, n-1, \ldots 1]=\mathrm{A}_{\text {inter }}\left(i^{\prime}, 1\right)[n, n-$ $1, \ldots 1]$ or $\mathrm{A}_{\text {inter }}(i, 1)[n, n-1, \ldots 1]=\mathrm{A}_{\text {short }}(j, 1)[n, n-1, \ldots 1]$ for some $i \neq i^{\prime}$.

Lemma 3. If $f_{\text {cond }}^{\prime}$ (see definition 7 ) returns 1 then at least one of the above four types of bad events occurs.

Proof. The proof is immediate.

Note that for a short query we add one element to $\mathrm{A}_{\text {short }}$ and for a long query we add $\ell=\ell(M)$ elements to $\mathrm{A}_{\mathrm{int}}$. In total we update $\sigma$ elements in two databases after $q$ queries, where $\sigma$ is the total number of blocks in all $q$ queries (both short and long). We define bad to be one of the Bad events when we add $i$ th element, $1 \leq i \leq \sigma$. The complement of the event is denoted by good ${ }^{i}$. We estimate the following probability for different possible cases:

$$
\operatorname{Pr}\left[\operatorname{bad}^{i} \mid \wedge_{j=1}^{i-1} \operatorname{good}^{j}\right] .
$$

We divide Bad events into two cases based on whether the $i$ th update $(u, v)$ is on $\mathrm{A}_{\text {short }}$ or on $\mathrm{A}_{\text {inter }}$.

- Case 1. Bad event on the update of $A_{\text {short }}$ : It can happen in two ways. Either the adversary correctly guesses $u$ which already exists in $A_{\text {inter }}$ or the outputs collide accidentally with one of the previous outputs stored in $A_{\text {short }}$ or $A_{\text {inter }}$ given that the guess is not correct. Note that if the guess is not correct then the input $u$ is fresh and its output is uniformly distributed. The collision occurs in one of the $n$-bits with probability at most $2(i-1) / 2^{n}$. Moreover, if $u$ 
appears as $j$ th intermediate input of $F W P^{\mathrm{ro}}(M)$ for some $M$ such that $(M, j)$ corresponding to an element of $A_{\text {inter }}$ then the type- 4 bad event occurs with probability $(i-1) / 2^{n}$.

Now, given that good event, all information to $\mathcal{A}$ so far, is independent of the internal computation. So the guess is correct with some internal input having the probability bounded by $(i-1) / 2^{n}$. So

$$
\operatorname{Pr}\left[\operatorname{bad}^{i} \mid \wedge_{j=1}^{i-1} \operatorname{good}^{j}\right] \leq 4(i-1) / 2^{n} .
$$

- Case 2. Bad event on the update of $A_{\text {inter }}$ : This probability can be bounded by random oracle collision probability as the input $u$ freshly appears due to the good event. The following can be shown easily:

$$
\operatorname{Pr}\left[\operatorname{type}-4 \text { bad }^{i} \mid \wedge_{j=1}^{i-1} \operatorname{good}^{j}\right] \leq(i-1) / 2^{n}, \operatorname{Pr}\left[\operatorname{type}-2 \text { or } 3 \operatorname{bad}^{i} \mid \wedge_{j=1}^{i-1} \operatorname{good}^{j}\right] \leq 2(i-1) / 2^{n}
$$

and hence $\operatorname{Pr}\left[\operatorname{bad}^{i} \mid \wedge_{j=1}^{i-1} \operatorname{good}^{j}\right] \leq 3(i-1) / 2^{n}$.

Combining all these cases we obtain that the probability of bad event is at most $\sigma(\sigma-1) / 2^{n-1}$. Now we state our indifferentiability results.

Theorem 4. The FWP hash is $\left(t_{\mathcal{A}}, t_{S}, q, \sigma, \varepsilon^{*}\right)$-indifferentiable in the random oracle model for the compression function, for any $t_{\mathcal{A}}$, with $t_{S}=\ell \cdot \mathrm{O}\left(q^{2}\right)$ and $\varepsilon^{*}=\sigma^{2} / 2^{n-1}$ where the simulator $S$ is described in Algorithm 5.3 .

\section{Resistance of FWP Against Some Recent Attacks}

One of the most significant works in hash function cryptanalysis in recent times is the discovery of the multi-collision attack on the Merkle-Damgärd mode [11. Using similar technique as multi-collision attack, Kelsey and Schneier devised another very influential attack that recovered 2nd preimage with work lower than the brute-force when long messages were used in the MerkleDamgärd mode. These two attacks do not work on the FWP mode. Any variants of these types of attacks do not seem to work too on the FWP transform. The above two attacks crucially rely on the intermediate collisions on $n$-bit chaining values which cannot be adjusted by message modification. The FWP mode has $2 n$-bit chaining value which also cannot be adjusted by message modification. Therefore, the complexity of such attacks on the FWP mode appears to be no less than the bruteforce. The same argument applies to the FWP's resistance to Herding attack [12] too. In the full version of the paper we shall provide further evidence why the FWP should be able to resist all variants of the above attacks.

\subsection{Comparison of the FWP with Other Modes}

The highlight of the FWP mode is that the compression function takes $n$ bits of previous chaining value while produces $2 n$ bits of ouput. With the emergence of new types of attacks on the MerkleDamgärd mode (see Sect. 6), it has been found necessary that the compression function output should be at least $2 n$ bits to generate $n$ bits of hash output. This type of constructions is known as the Wide-pipe mode propounded by Lucks [15] (see Fig. 4 (c)). Many modern hash functions use this type of mode [9] to defend against multi-collision type attacks. The main problem with that mode is that the $2 n$ bits of chaining value, which are fed into the next compression function, reduce the bandwidth of the message-block and, thereby, impede the speed of the hash function. To 
skirt this difficulty the Sponge construction with $2 n$ bits of compression function output has been proposed [2] (see Fig. 4(d)). Unfortunately this construction collapses as easily as Merkle-Damgärd mode against all the attacks of Sect. 6. Another competing proposal is the HAIFA [5] mode. The HAIFA mode can be viewed as a special Merkle-Damgärd mode with an additional counter injected into each compression function call. This extra counter is very useful to thwart the attacks described in 13 12. However, the price to pay is the reduction of bandwidth for message in each compression function call, resulting in slower performance. In addition, the HAIFA mode is still as weak against Joux's multi-collision attack as the old Merkle-Damgärd mode.

\section{Conclusion and Open Problems}

This paper proposes a new sequential mode of operation, known as FWP, to hash messages of arbitrary length. The mode is collision-resistance-preserving, preimage-resistance-preserving and indifferentiable from a random oracle up to $\mathcal{O}\left(2^{n / 2}\right)$ compression function invocations. The mode is also shown to be more efficient than the Wide-pipe mode. Comparison of the FWP with other proposals has been outlined. No known attacks have so far been found in this mode, indicating that it may be possible to stretch the indifferentiable security bound of the mode beyond the birthday barrier of $2^{n / 2}$. We leave this as an open problem.

\section{Acknowledgements}

The first author was supported in part by the National Science Foundation, Grant CNS-0937267.

\section{References}

1. Mihir Bellare and Thomas Ristenpart. Multi-Property-Preserving Hash Domain Extension and the EMD Transform. In Xuejia Lai and Kefei Chen, editors, ASIACRYPT 2006, volume 4284 of Lecture Notes in Computer Science, pages 299-314. Springer, 2006. (Cited on pages 2 and 6)

2. Guido Bertoni, Joan Daemen, Michael Peeters, and Gilles Van Assche. On the Indifferentiability of the Sponge Construction. In Nigel P. Smart, editor, EUROCRYPT, volume 4965 of Lecture Notes in Computer Science, pages 181-197. Springer, 2008. (Cited on pages 2 and 15)

3. Rishiraj Bhattacharya, Avradip Mandal, and Mridul Nandi. Indifferentiability Characterization of Hash Functions and Optimal Bounds of Popular Domain Extensions. Indocrypt 2009. (Cited on page 12 )

4. Rishiraj Bhattacharyya, Avradip Mandal, and Mridul Nandi. Security Analysis of the Mode of JH Hash Function. In Seokhie Hong and Tetsu Iwata, editors, FSE, volume 6147 of Lecture Notes in Computer Science, pages 168-191. Springer, 2010. (Cited on page 2,)

5. Eli Biham and Orr Dunkelman. A framework for iterative hash functions - HAIFA. Second NIST Cryptographic Hash Workshop, 2006, 2006. (Cited on pages 2 and 15)

6. Gilles Brassard, editor. Advances in Cryptology - CRYPTO '89, 9th Annual International Cryptology Conference, Santa Barbara, California, USA, August 20-24, 1989, Proceedings, volume 435 of Lecture Notes in Computer Science. Springer, 1990. (Cited on pages 15 and 16.)

7. Jean-Sébastien Coron, Yevgeniy Dodis, Cécile Malinaud, and Prashant Puniya. Merkle-Damgård Revisited: How to Construct a Hash Function. In Victor Shoup, editor, CRYPTO 2005, volume 3621 of Lecture Notes in Computer Science, pages 430-448. Springer, 2005. (Cited on pages 2 and 6)

8. Ivan Damgård. A Design Principle for Hash Functions. In Brassard [6], pages 416-427. (Cited on page [1) 
9. R. Rivest et al. The MD6 Hash Function. (Cited on page 14)

10. Shoichi Hirose, Je Hong Park, and Aaram Yun. A Simple Variant of the Merkle-Damgård Scheme with a Permutation. In Kaoru Kurosawa, editor, ASIACRYPT, volume 4833 of Lecture Notes in Computer Science, pages 113-129. Springer, 2007. (Cited on page 2])

11. Antoine Joux. Multicollisions in Iterated Hash Functions: Application to Cascaded Constructions. In CRYPTO 2004, pages 306-316, 2004. (Cited on pages 2 and 14)

12. John Kelsey and Tadayoshi Kohno. Herding Hash Functions and the Nostradamus Attack. In Serge Vaudenay, editor, EUROCRYPT, volume 4004 of Lecture Notes in Computer Science, pages 183-200. Springer, 2006. (Cited on pages 2, 14 and 15.)

13. John Kelsey and Bruce Schneier. Second Preimages on n-Bit Hash Functions for Much Less than $2^{\mathrm{n}}$ Work. In Ronald Cramer, editor, EUROCRYPT 2005, volume 3494 of Lecture Notes in Computer Science, pages 474-490. Springer, 2005. (Cited on pages 2 and 15 )

14. A. Klimov and A. Shamir. New Cryptographic Primitives Based on Multiword T-Functions. In B. Roy and W. Meier, editors, Fast Software Encryption 2004, Lecture Notes in Computer Science, pages 1-15. Springer, 2004. (Cited on page 2,)

15. Stefan Lucks. A failure-friendly design principle for hash functions. In Bimal K. Roy, editor, $A S I-$ ACRYPT, volume 3788 of Lecture Notes in Computer Science, pages 474-494. Springer, 2005. (Cited on pages 2 and 14 )

16. Ueli M. Maurer, Renato Renner, and Clemens Holenstein. Indifferentiability, impossibility results on reductions, and applications to the random oracle methodology. In Moni Naor, editor, TCC, volume 2951 of Lecture Notes in Computer Science, pages 21-39. Springer, 2004. (Cited on pages 2 and 6 .)

17. Ralph C. Merkle. One Way Hash Functions and DES. In Brassard 6], pages 428-446. (Cited on pages 1 and 2)

18. NIST. Secure hash standard. In Federal Information Processing Standard, FIPS-180, 1993. (Cited on page 1 .)

19. NIST. Secure hash standard. In Federal Information Processing Standard, FIPS 180-1, April 1995. (Cited on page 1.)

20. R. Rivest. The MD4 message-digest algorithm. In A. J. Menezes and S. Vanstone, editors, CRYPTO, volume 537 of Lecture Notes in Computer Science, pages 303-311. Springer, 1990. (Cited on page 1)

21. R. Rivest. The MD5 message-digest algorithm. In IETF RFC 1321, 1992. (Cited on page 1)

22. David Wagner. A Generalized Birthday Problem. In Moti Yung, editor, CRYPTO, volume 2442 of Lecture Notes in Computer Science, pages 288-303. Springer, 2002. (Cited on page 5])

23. Hongjun Wu. The JH Hash Function. The 1st SHA-3 Candidate Conference. (Cited on page 2,)

\section{A Comparison of Modes}

\section{B Arbitrary Distinguisher}




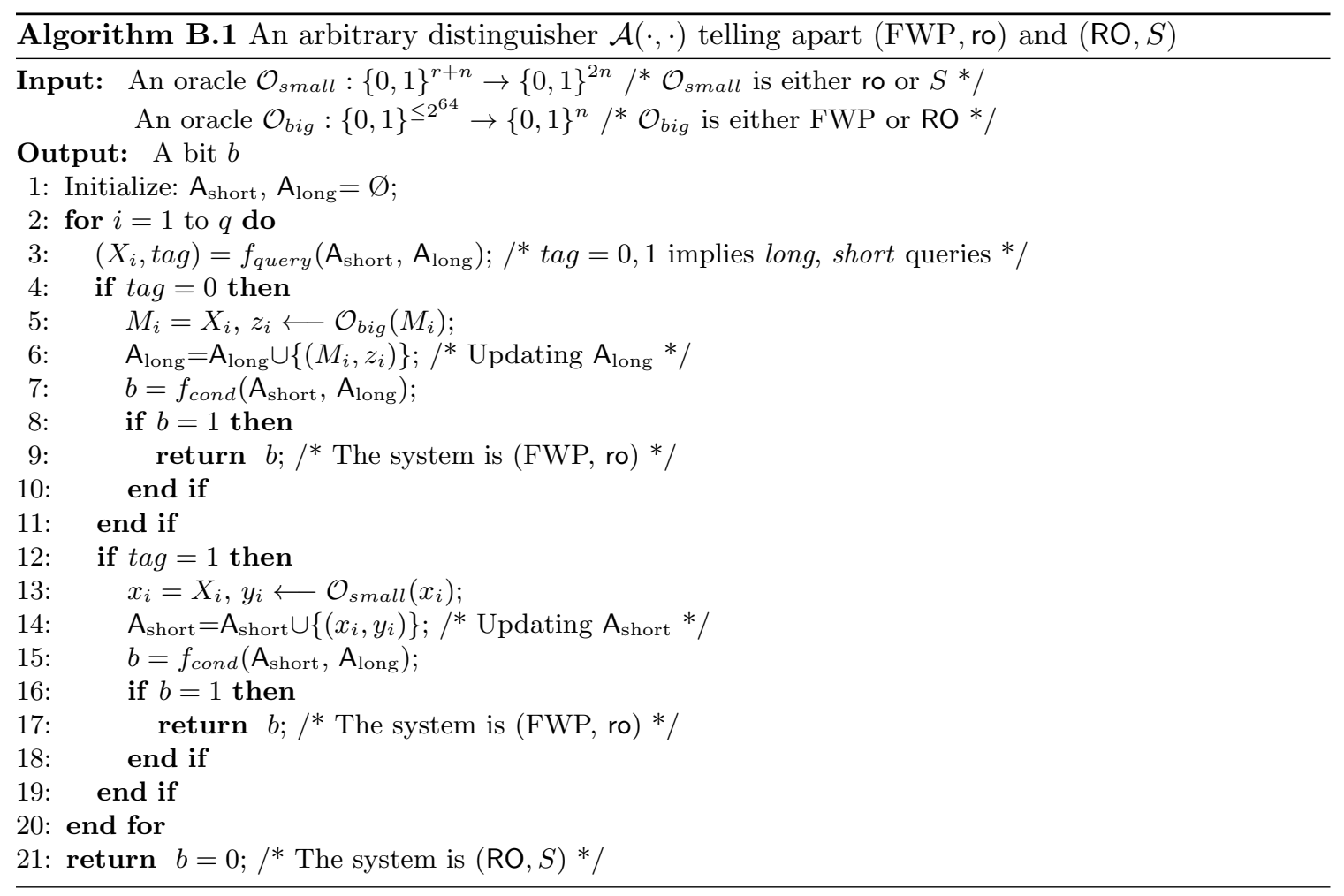




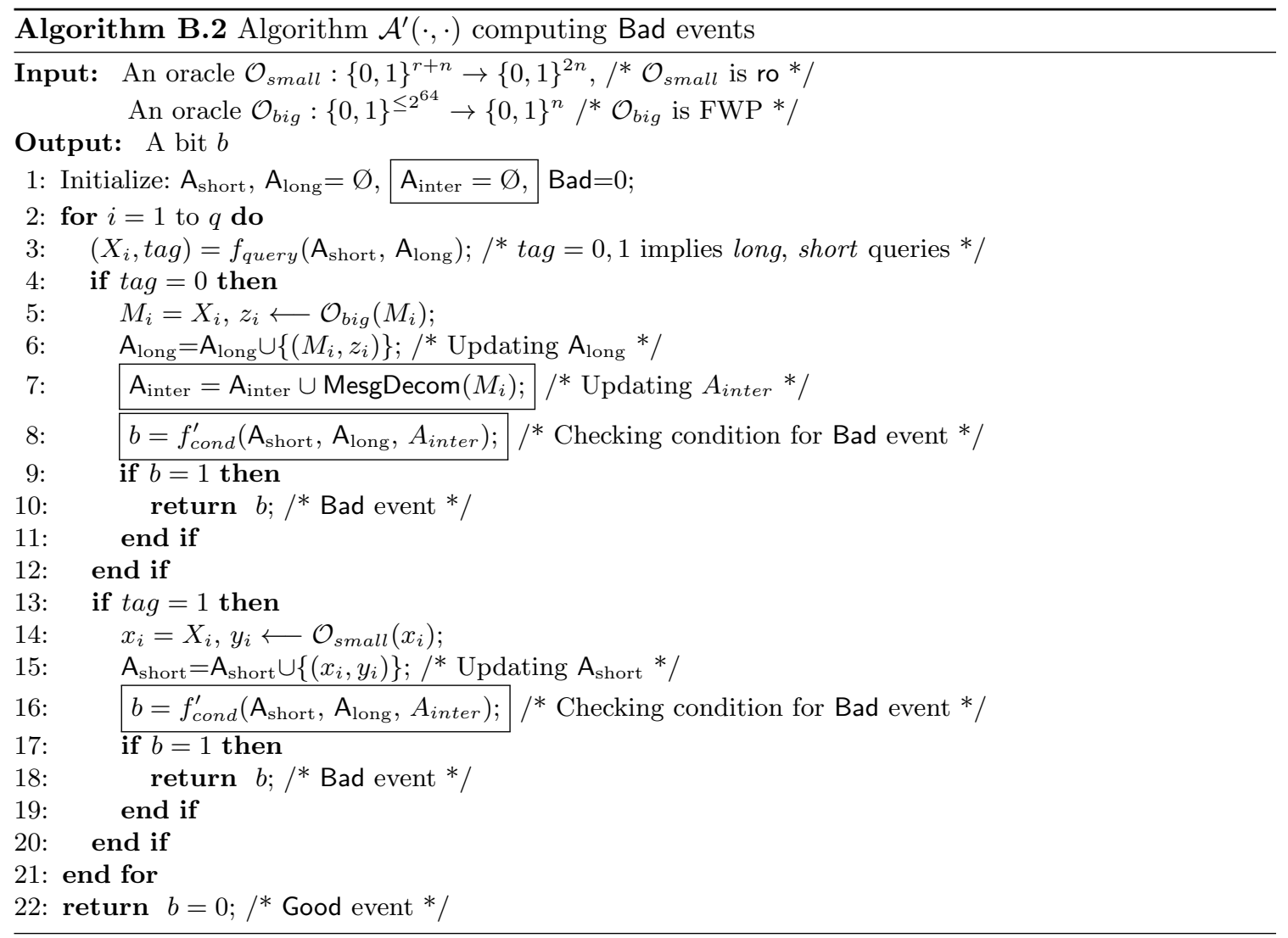




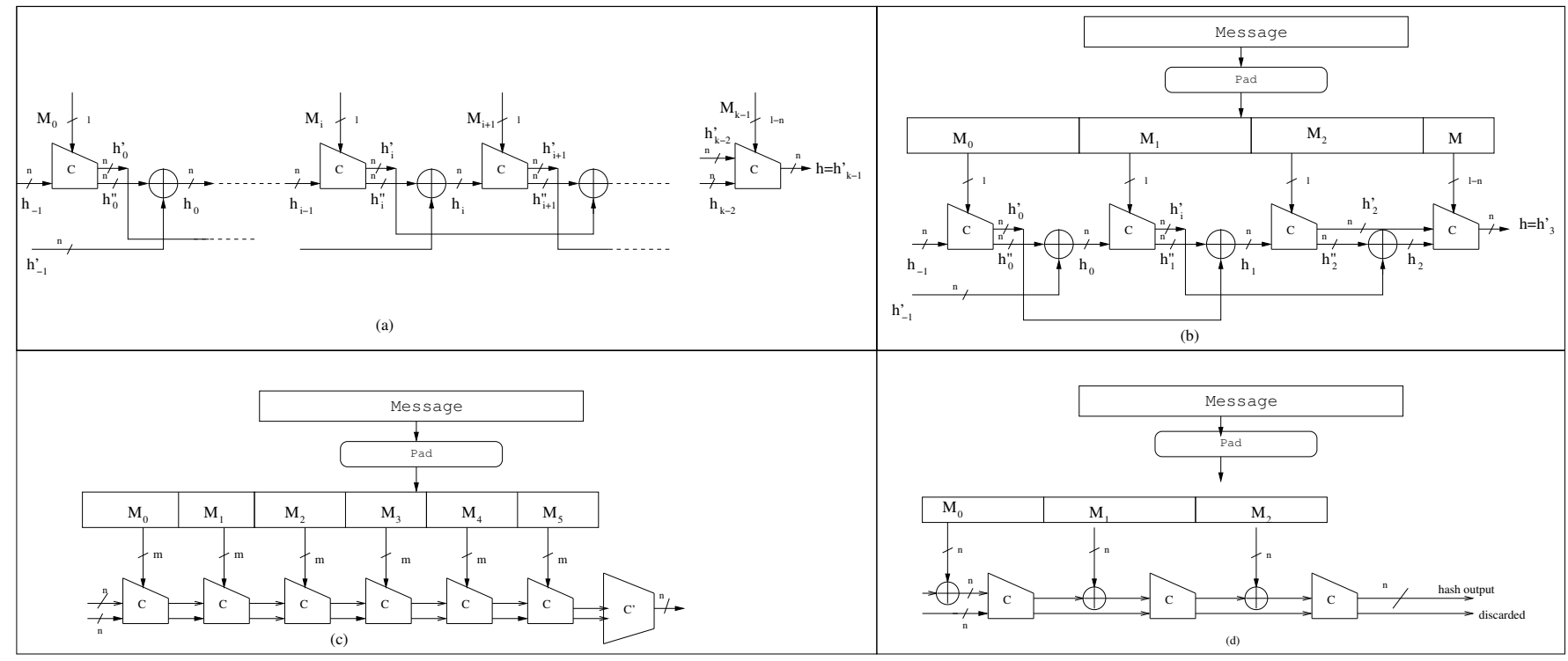

Fig. 4. (a) The FWP mode. (b) A four-block example of the FWP mode. (c) The Widepipe mode. (d) The Sponge mode. 